\title{
Late lumen enlargement and plaque regression after drug-coated balloon treatment for an isolated ostial lesion of a diagonal branch
}

\author{
Song Lin Yuan ${ }^{1,2}$, Eun Jung Jun ${ }^{1}$, Moo Hyun Kim², Eun-Seok Shin ${ }^{1}$ \\ ${ }^{1}$ Department of Cardiology, Ulsan Medical Center, Ulsan, South Korea \\ ${ }^{2}$ Department of Cardiology, Dong-A University Hospital, Busan, South Korea
}

A 55-year-old man was admitted with worsening effort angina for over 1 week. Hypertension was the only coronary risk factor in this patient, and resting electrocardiography and echocardiography findings, as well as cardiac enzyme levels were normal. Coronary angiography revealed ostial stenosis of the first diagonal branch (90\% diameter narrowing) (Fig. 1A, Suppl. Video 1). The patient declined complex stent implantation but agreed to receive drug-coated balloon (DCB) treatment. Informed consent was obtained, and the patient underwent careful evaluation.

The ostium of the diagonal branch was dilated several times using a $3.0 \times 13 \mathrm{~mm}$ scoring balloon at a pressure of $10 \mathrm{~atm}$, followed by placement of a DCB $(3.0 \times 20 \mathrm{~mm})$, which was inflated to a pressure of $8 \mathrm{~atm}$ for $60 \mathrm{~s}$. Final angiography revealed no significant dissection or residual ostial stenosis of the diagonal branch and the main vessel of the left anterior descending artery (Fig. 1B, Suppl. Video 1). Intravascular ultrasound revealed increased luminal area with plaque dissection at the ostium of the diagonal branch (Fig. 1B4, arrow), and the patient's angina was resolved. Nine-month follow-up angiography (Fig. 1C, Suppl. Video 1) and intravascular ultrasound findings confirmed excellent results with plaque reduction (Fig. 1C1-C5) and a healed dissected ostial plaque (Fig. 1C4).

Percutaneous coronary intervention for isolated ostial lesions is challenging. It was observed that DCB treatment resulted in a significant reduction in the plaque burden with ostial lumen enlargement without any left anterior descending artery compromise. These findings suggest that DCB treatment may potentially be indicated for ostial lesions, particularly in patients who refuse to undergo stenting.

Conflict of interest: None declared

Address for correspondence: Eun-Seok Shin, MD, PhD, Department of Cardiology, Ulsan Medical Center, 13, Wolpyeong-ro, 171 beon-gil, Nam-gu, Ulsan, 44686, South Korea, tel: +82-52-259-5020, fax: +82-52-259-5120, e-mail: sesim1989@gmail.com 


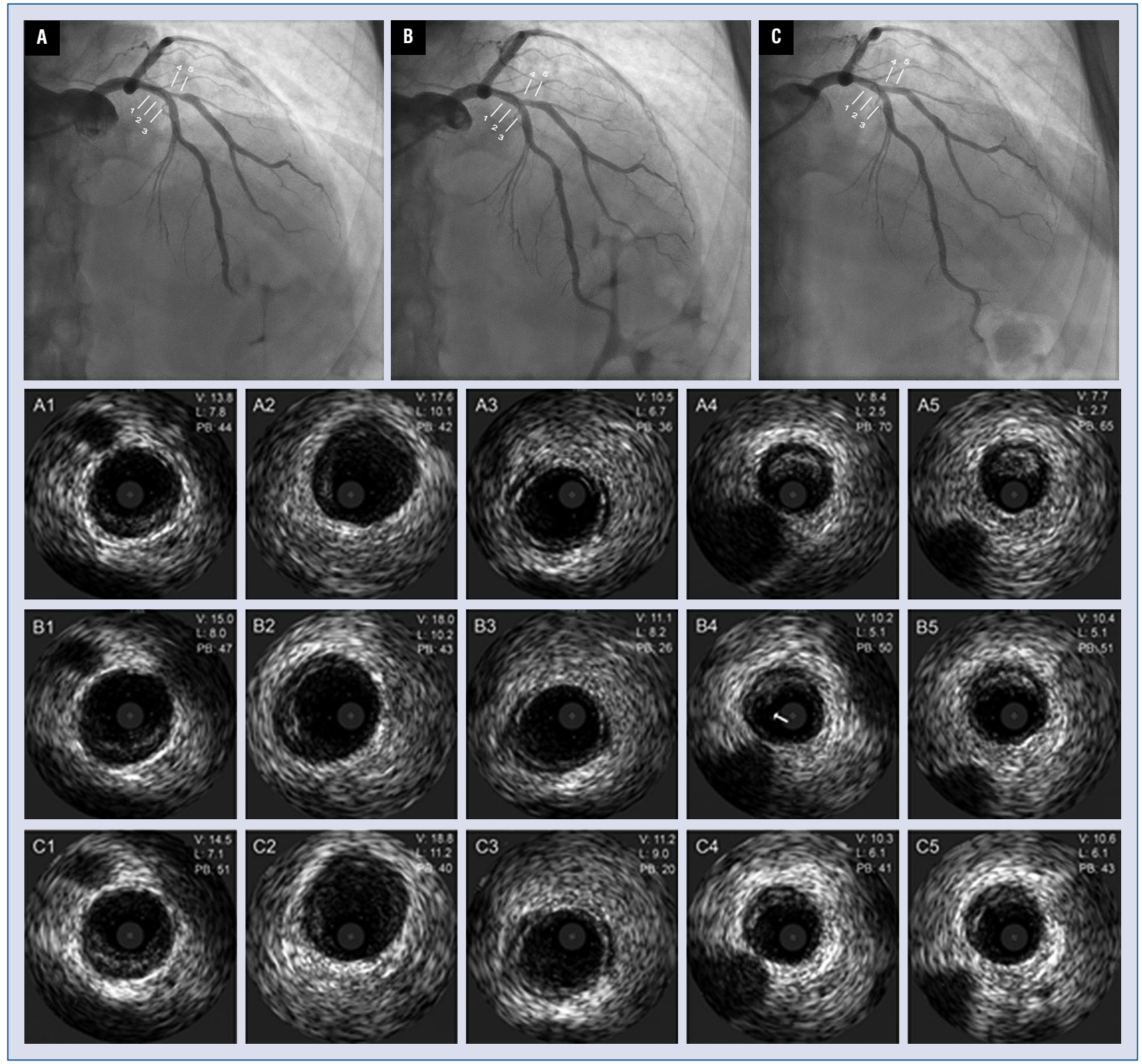

Figure 1. Pre-procedure images (A), post-drug-coated balloon treatment images (B), follow-up angiographic images (C), and those coupled with serial corresponding intravascular ultrasound images. Nine-month follow-up intravascular ultrasound showing significantly increased luminal area with a decreased plaque area at the ostium of the diagonal branch without a significant change in the main vessel of the left anterior descending artery; $\mathrm{V}-$ vessel area $\left(\mathrm{mm}^{2}\right)$; $\mathrm{L}$ - lumen area $\left(\mathrm{mm}^{2}\right)$; $\mathrm{PB}$ - plaque burden (\%). 